\title{
Word Production (Al-Tawlīd Al-Lugawy) in Arabic: Causes and Types
}

\section{التوليد اللغويّ في اللغة العربية: أسبابه وأنواعه}

\section{Uki Sukiman}

uki.sukiman@uin-suka.ac.id

Department of Arabic Language and Literature

UIN Sunan Kalijaga Yogyakarta

• Received: 07.10.2020 • Accepted: $30.04 .2021 \quad \bullet$ Published online: 05.05.2021

Abstract: Word Production (Called in Arabic by Al-Tawlìd Al-Lugawy) which means inventing new words is one of development of language phenomenon that happens in any languages including Arabic. This phenomenon is a demand of condition that one language is not capable of expressing ideas and emotions and has something to do with communication with non-speaker or other languages. Thus, a language can influence and be influenced. This paper tried to uncover reasons and theories using comparative study so that it is clear for Arabic language researchers to use tawlid in their research. The writer found eight reasons why neology emerged, which are development of civilization, friction between Arab and Non-Arab, administration system after Islamic expansion, translation process from Greek into Arabic, social and political demands, the beauty of expression, and inventing new scientific terms, while Ibrahim Murad stated five theories about neology: phonetic neology, morphological neology, semantic neology, spontaneous neology, and borrowing neology; as Nasruddin suggested five theories such as transfer of meaning, derivation, derivation from Arabization (mu'rab) or loanwords (dakhil), taulid form coinase and structure, metaphor neology, and meaning expansion.

Keywords: word production, arabic Language, arabization, loanwords

$$
\text { الملحصبية: التوليد بمعنى إحداث كلمة جديدة مظهر من مظاهر تطوراللغات منها اللغة }
$$




\section{Uki Sukiman}

اللغة و مشاعرهم خاصة و نتيجة من تعاملهم مع الآخرين فأثروا و تأثّروا. اهتمّ كثير من علماء

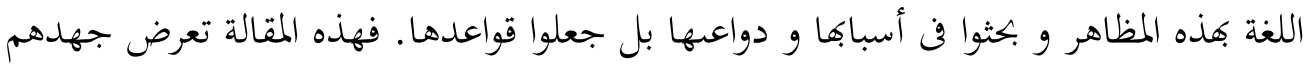
فن هذا المجال فن اكتشاف تلك أسباب والقواعد بمنهج مقارنة حتى تكون واضحة لمببي اللغة العربية راجيا استعمالهمم في بحوثهم و مقالتهم.وجد الكاتب ثمانة أسباب لظهور التوليد في اللغة العربية وهي التطور الحضاري، والاحتكاك بين العرب الأعجم، والحاجة الملحة، والنظام الإداري عند العرب بعد الفتوحات الإسلامية، وعملية ترجمة العلوم اليونانية إلى اللغة العربية، والدعاية السياسية والاقتصادية، وجمال التعبير، ووضع المصطلحات العلميةكما وجد ابراهيم مراد الذي قدم خمس قواعيد يعني التوليد الصوتي، التوليد الصرفي، التوليد الدلالي، التوليد بالارتحال، التوليد بالانقراضحيث لكل منها فصول كما قدم نصر الدين ست قواعيد وهي

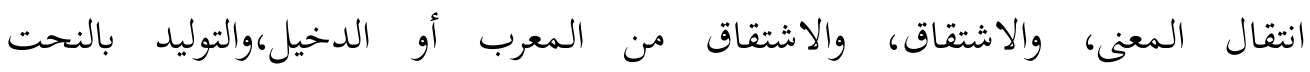
والتركيب،والتوليد المجازي، والتوسع. كلمات دلالية: التوليد ، اللغة العربية، تتطوّر اللغة، الاشتقاق.

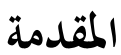

اللغة هي أداة اتصال لفهم بعضنا البعض. تعمل اللغة كوسيلة لنقل أفكار ومشاعر ورغبات المتحدثين من البشر. لا يوجد نشاط بشري واحد بصرف النظر عن دور اللغة. واللغة

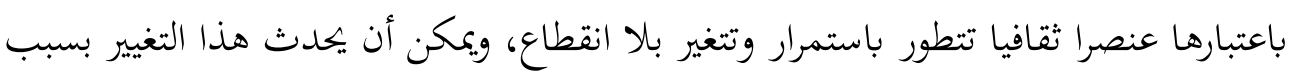
متطلبات العصر أو الاحتكاك مع اللغات الأخرى. بحيث يمكن أن تؤثر لغة واحدة على لغات أخرى ويمكن أن تتأثر هذه اللغة أيضا باللغات الأخرى. فاللغة ليست ثابتة ولكنها ديناميكية. والقول بأن اللغة ديناميكية لأهما تتغير وفقا للسياق وراءها، وتحدث هذه التغييرات على هلى مستويات مختلفة تتراوح بين علم الأصوات، والصرف والنحو، والدلالة والمعجم. ومن بين كل هذه المستويات، كان التغير الأكثر وضوحا هو التغير المعجمي Chaer and Agustina) (2010. لقد شهد مستوى المعجم العديد من التطورات، وذلك بسبب ظهور كلمات تأتي من لغات أخرى وتتحول وتصبح ضمن مفردات لغة معينة. 


\section{Uki Sukiman}

وفي هذا الصدد، تظهر دراسة لغوية تناقش التغييرات في المفردات الجديدة تسمى بعلم التوليد اللغوي أو المولد. يشير علم التوليد اللغوي إلى استخدام مفردات جديدة أو استخدام معاني جديدة للمفردات الموجودة. قد تتقاطع دراسة علم الحديث مع دراسات أخرى في اللسانيات وهي دراسة الدخيل والتعريب. فالدخيل هو اللفظ الأعجمي الذي أدخل كلام

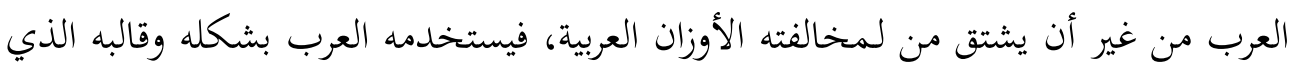
دخل العرب، فكلمة "كلاسيك"،على سبيل المثال، تدخل ضمن موضوع الدخيل، لأهماليستمن الكلمات العربيةالقياسية من حيث الوزن أو الاشتقاق. أما التعريب فهو اللفظ الذي دخل العربية، وعومل معاملة اللفظ العربي من حيث الوزن والاشتقاق. كلمة "ديوان"

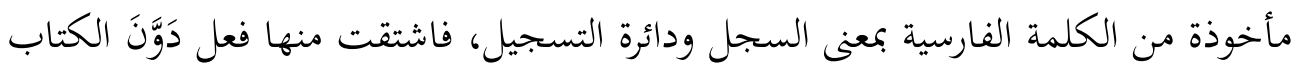
أو الأسماء وهو مدون (اسم فاعل) (Tunji 2005).

نستطيع القول إن التوليد يعد مظلة للتغير اللغوي؛ التعريب والدخيل، أي هما جزءان التوليد وليسا العكس. وهذه المصطلحات الثلاثة هي أشكال لعملية التغير اللغوي الشائع في اللغة العربية. والصورة أدناه توضح أن التغير اللغوي، من حيث الشكل والمعنى، يمكن أن يمر عبر ثلاث عمليات؛ علم التوليد اللغوي والتعريب والدخيل. نستطيع القول إن التوليد يعد مظلة للتغير اللغوي؛ التعريب والدخيل، أي هما جزءان التوليد وليسا العكس. وهذه المصطلحات الثلاثة هي أشكال لعملية التغير اللغوي الشائع في اللغة العربية. والصورة أدناه توضح أن التغير اللغوي، من حيث الشكل والمعنى، يمكن أن يمر عبر ثلاث عمليات؛ علم التوليد اللغوي والتعريب والدخيل. 


\section{Uki Sukiman}

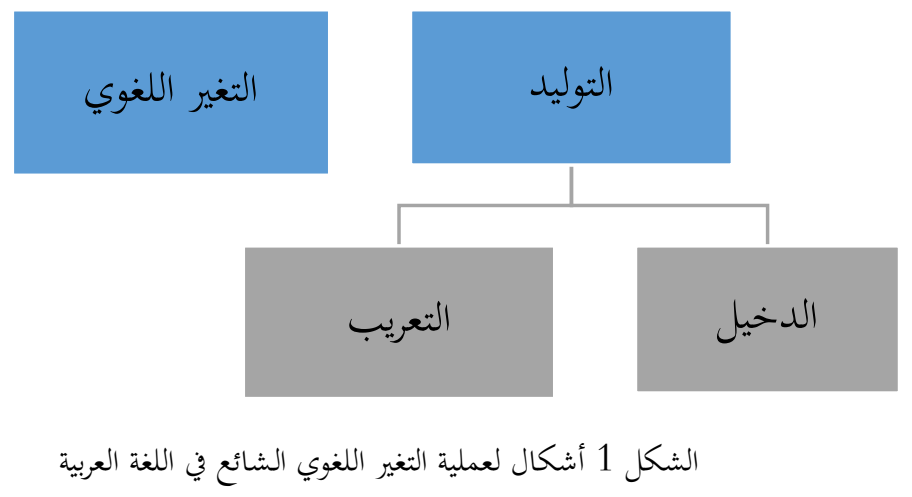

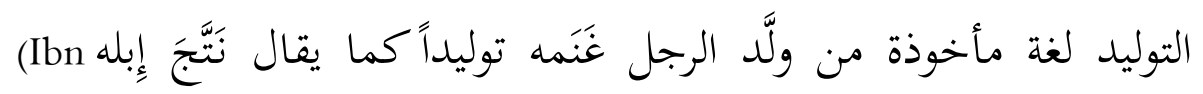
(Mandur, n.d.) الاصطلاح يرى بعض اللغويين العرب مثل ما ورد في أمالي ثعلب: سُئل عن التغيير:

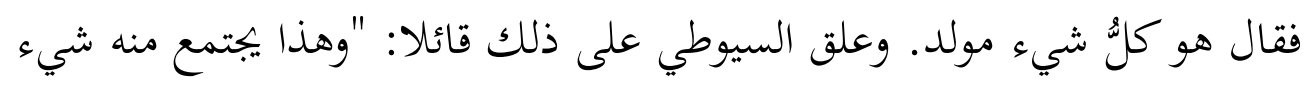

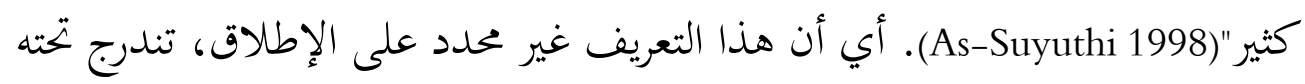

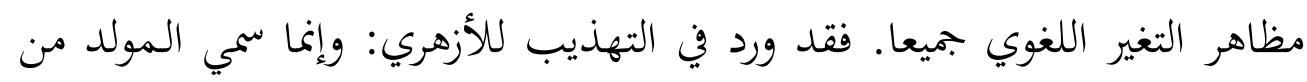
الككلام مولدا إذا استحدثوه ولم يكن من كلامهم فيما مضا (Al-Azhari 2001).

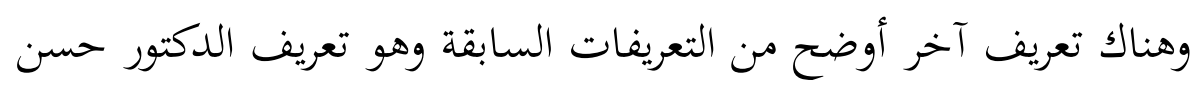

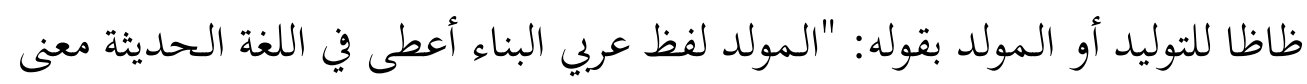

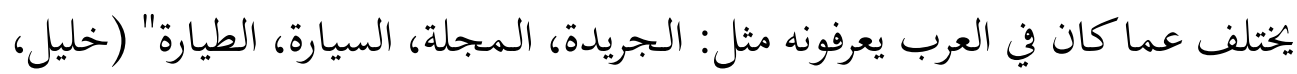

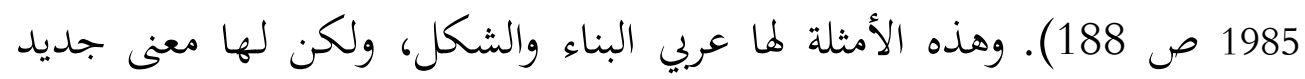

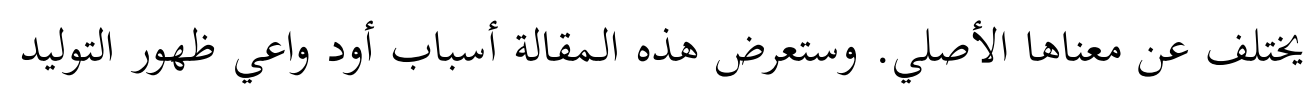

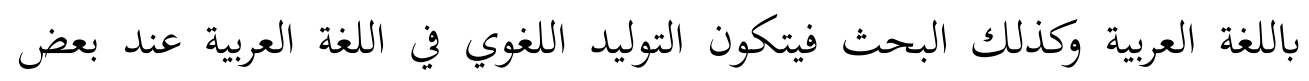

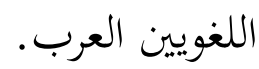

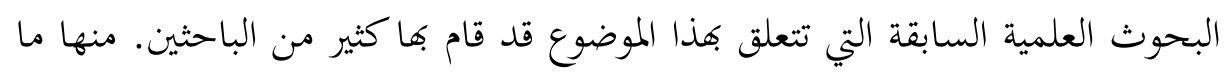

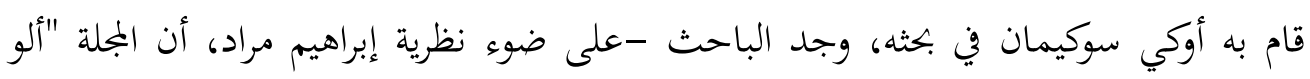




\section{Uki Sukiman}

إندونيسيا" تستخدم التوليد الدلالي والتوليد بالاقتراض أكثر من الآخر (Sukiman 2020). أكبر المشاكل في عملية الترجمة الإندونيسية إلى العربية هو أنه ليس من المصوتة والصامتة موجودة في اللغة العربية ومشكلة البحث عن الفكرة الأنسب في التعبير (Sukiman 2020). كتبت بلقيس أمين الله نور المفتاح بحثا عن إحداث كلمة جديدة في اللغة العربية في مجال تكنولوجيا وخصت الجريدة "الأخبار" كموضوع بحثه (Mivtakh 2019). بالإضافة إلى ذلك، البحث حول هذا المجال قامت به أيضا أسنا أندرياني طالبة في الدراسات العلي بجامعة سونان كاليجاكا جوكجاكرتا (Andriani 2010). كتبت أسنا في رسالتها التوليد والتعريب والتحليل الصرفي والتحليل والصوتي الذي يوجد في المعجم المورد. التوليد والتعريب في إحداث مفردان جديدة في وسائل التواصل الاجتماعي واتسآب وفيسبوك قد سبق بحثه من قبل حنون خيرة النساء مع أن أولياء نبيلة

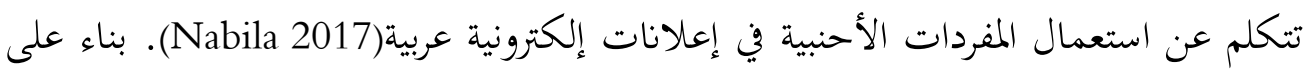
البحوث السابقة، إنه قابل بالقوب بأن هناك كثير من البحوث العلمية التي تبحث فيها عن

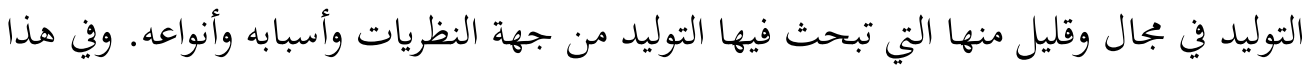
الطريف هذا البحث مهم القيام به.

\section{منهجية البحث}

يتركز هذا البحث على مظهر من مظاهر التوليد في اللغة العربية عامة و على أراء علماء العرب فن مواجهة هذه المظاهر خاصة. فليس موضوع هدا البحث اجماع كلمة أو كلمات دخلت فن اللغة العربية ولكن يهتمّ البحث بمقارنة أراء العماء حول التوليد فن هذه اللغة. فمنهج المقارنة هو منهج مناسب فن هذا البحث وهو منهج يقارن بين المعلومات حتى يتبين منه وجوه

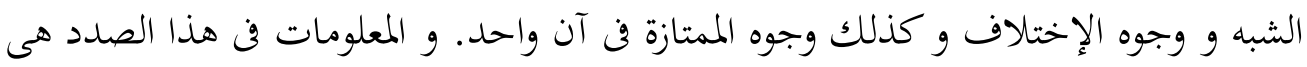
كتب تبحث في طريقة التوليد للغة العربية من اللغة الاخرى.

و الخطوات سيجرى بها الكاتب منها : 1) اجماع المعلومات أى الكتب أو المقالات التى

تتعلق هذا البحث، 2) وصف المعلومات وصفا دقيقا و تفصيلها تفصيلا جزئيا حتى يكون كلّ فصل أو جزء شيئاواضحا، 3) تحليل البيانات و مقارنتها بعضها بعض حتى يتضح لنا نواحها 


\section{Uki Sukiman}

المساوية و المتفارقة و يسهل للباحثين أو طلاب الجامعة أو المهتمين بتنمية اللغة العربية للتطبيقها

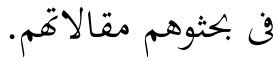

\section{نتائج الدراسة وتحليلها أسباب ظهور التوليد اللغوي}

يرى محمد عيد إلى لفظ "المولد" أطلق أولا على الأشخاص الذين وجدوا بين العرب الخلص ثم اتسع استعماله فأطلق على الكلام المحدث الذي ليس من أصل لغة العرب، وإنما هو كلام جديد شاع في المجتمع العربي مع ازدياد مخالطة الأجانب(Aid 1980'). وهذه المخالطة بالتأكيد لا تمثلا لدافع أو السبب وراء ظهور التوليد، هنا كأسباب أخرى لاتقل أهمية،و تؤثر على ظهور التوليد في اللغة العربية. تنوعت الأسباب التي دعت إلى وجود التوليد اللغوي ما بين أسباب حضارية واجتماعية وعلمي، غير أها لم تكن على درجة واحدة من الأثر في توليد الألفاظ، وتتمثل هذه الأسباب

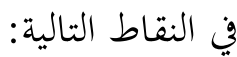

$$
\text { التطور الحضاري }
$$

هنا كالعديد من الكلمات التيتد خلفي حياة المسلمين عن طريق التوليد لتسد نقصا لغويا في ألفاظ الحضارة العبية. فمنذ نشأة المجتمع الإسلامي، هنا كعدة كلمات أو ألفاظ

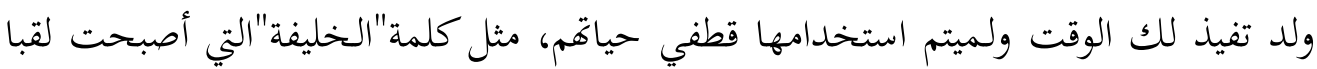
أطلق على أبي بكر باعتباره خليفة للنبي محمد. بعد ذلك ظهر لفظ جديد مثل"أمير المؤمنين"، وهو لقب عمر بن خطب بعد توليه الخلافة(Khalil 1985a).

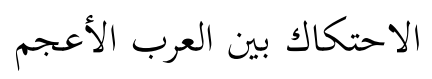

انتشر الإسلام في جميع أنحاء البلاد وحتى خارج شبه الجزيرة العربية وانتشر في يد يد

العلماء العرب. لميد مطويلا حتى دخل العديد من الأجانب غير الناطقين بالعربية إلى الإسلام.

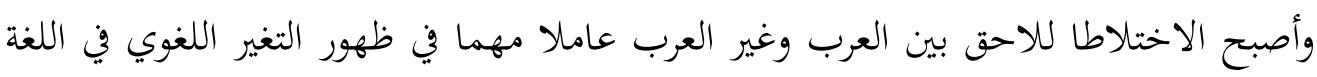




\section{Uki Sukiman}

العربية. ويشمل هذا التغير على مجالات الاقتصاد والصناعة والزراعة والتجارة والعلوم والفلسفة والدين وماإلى ذلك. الحاجة الملحة

هناك نوع من التغير في الدلالة يكون وليد الحاجة الملحة للتجديد في التعبير أو التسهيل في الفهم عند المخاطب. ويتم هذا عادة على يدي أصحاب المهارة في الكلام كالشعراء والأدباء لتوضيح الدلالة أو تقوية أثرها في الذهن. ووجد الإنسان مضطرا إلى التطور أيضا في الألفاظ المعبرة عن أدواته ومواصفاته وصناعاته وملابسه وأبنيته، فلجأ إزاء هذه الضرورة إلى التوليد الدلالي بأن يعمد إلى الألفاظ القديمة ذات الدلالات المندثرة فيحي بعضها، ويطلق على مستحدثاته متلمسا في هذا أدنى ملابسة (Anis 1958).

$$
\text { النظام الإداري عند العرب بعد الفتوحات الإسلامية }
$$

كان هذا النظام الذي أخذ به العرب بعد الفتح حافزا كبيرا لتوليد بجموعات عديدة من الألفاظ التي لم يعرفها العرب. وفي نطاق مناصب الدولة نجد ألفاظا جديدة مثل: الدولة، الشرطة، النقابة، الحجابة، ديوان، القضاء وغيرها.

و في المجال العسكري نجد: المسترزقة، المتطوعة، العسكر، كتيبة، وغيرها. وفي مجال الإدارة نجد: الثغور، العواصم، الولاية، والحكومة، الكاتب، وغيرها. وأما في نطاق المال الضرائب نجد: بيت المال، الخراج، الجزية ، وغيرها (Khalil 1985b). عملية ترجمة العلوم اليونانية إلى اللغة العربية

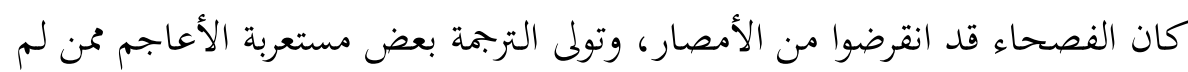
تستحكم مرةهم في العربية، فعزوا عن ترجمة بعض الألفاظ الأعجمية مع وجود مرادف لها في

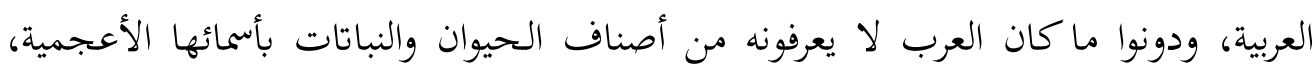
واستعمل فلاسفة الإسلام وأطباؤهم هذه الألفاظ، وخاصة من كان منهم من سلالات أعجمية، كالفرابي والرازي وبن سينا (Wafi 2014). وتحدث الدكتور إبراهيم نجا كما أشار إليه نصر الدين

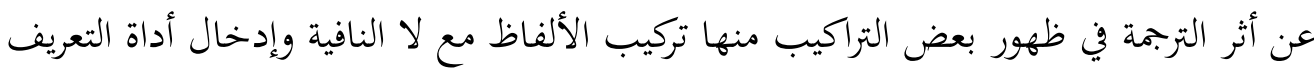




\section{Uki Sukiman}

عليها مثل اللا هاية واللا ضرورة. وكذلك صوغ الاسم من الحروف او الضمائر كالكمية والكيفية .(Anis 1958)

$$
\text { الدعاية السياسية والاقتصادية }
$$

قد تكون الدعاية السياية أو الاقتصادية دافعا كبيرا لتوليد الألفاظ الجديدة. فأصحاب وله الإعلانات التجارية يقومون بتخير الألفاظ رغبة في رواج بضائعهم وأسواقهم. فصاحب محل المشروبات قد يطلق على محله "جنة الفواكه" والحلاق يطلق على دكانه "دار الزينة"، الخياط قد يقول عن محله دار الأناقة" والطورشجي قد يدعو ما يبيعه "بالمشهيات" وغير ذلك مما هو مألوف لنا في حياتنا العامة (1958 (Anis).

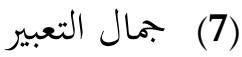

التوليد الذي يشير إليه خليل(Khalil 1985a) هو توليد اصطلاحات مركبة من الكلمات التي تدل بطريقة بجازية على بعض المعاني الخاصة، مثال ذلك: استنعجب الذئاب للعدو يبدي الصداقة، وضرب الرقم السياسي: ذهب بعيدا في النجاح أو الفشل.

$$
\text { وضع المصطلحات العلمية }
$$

قد تكون اللغةحمدودة - في بعض الحالات - في وضع المصطلحاتالعلمية، وكان السبيل إلى ذلك التوليد اللغوي. وقد لعب التوليد دورا مهما في وضع المصطلحات المتعلقة بالدين والعلوم اللغوية. وهذا ما أشار إليه حلمي خليل بقوله: "...لأن اللغة لم تعد لغة الشعر فقط، بل أصبحت أيضا لغة العلوم. ومن المصطلحات الجديدة : الثلاجة، والغسالة، والمذياع والمسجل والمكبر (Khalil 1985a). وبفضل هذه الأسباب المتنوعة تولد الألفاظ الجديدة المسماةب" المولدة". وتوضح هذه الألفاظ الجديدة أنماطا مختلفة للتوليد باللغة العربية.

$$
\text { 2. أنواع التوليد وطرقه }
$$

تنوعت طرق التوليد في العربية تنوعاكبيرا. قد ذكر مراد أن كل ما يولد في اللغة من المفردات إنما يولد بحسب ما تسمح به قواعدها نتيجة قوانين استعماها من الوسائل Murad)

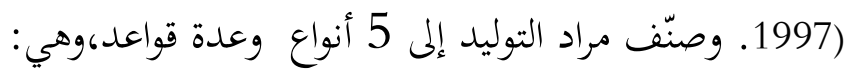




\section{Uki Sukiman}

1

وهو إحداث وحدات صوتية معجمية جديدة نتيجة ما يطرأ على المفردات من تغيير صوتي. وقواعد هذا التوليد خمس هي: أ. الإبدال، هو نزوع أحد الصوتين المتقاربين أو المتماثلين في المخرج أو في الصفة إلى أن

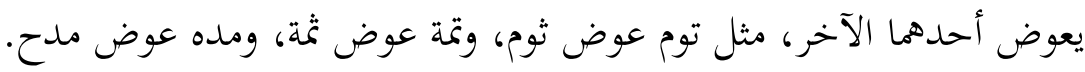

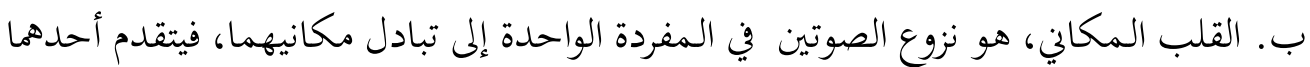

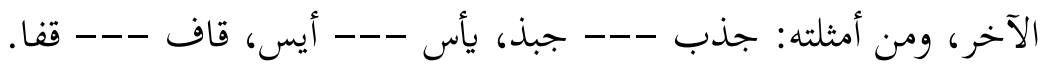

ت. نزوع الأصوات المتجاورة في المفردة الواحدة إلى تأثر بعضها ببعض عند النطق بها تأثرا يحدث عنه تقارب في الصفة أو في المخرج. وهو نوعان: أ) تماثل تقدمي، يكون بتأثر الصوت الثاني بالأول، ومن أمثلته في العربية ازتان --ازدان، اضتجع --- اضطجع.

ب) تماثل رجعي، يكون بتأثر الصوت الأول بالثاني، ومن أمثلته في العربية: سلخ --صلخ، سور --- صور .

ج) التباين، وهي نزوع الصوتين المتماثلين في المفردة الواحدة إلى التخالف، مثل لفظ أترج تحول إلى أتنرج، ولفظ قبرة تحول إلى قنبرة.

د) الإقحام، ويكون بإدخال صوت غير أصلي في تأليف الوحدة المعجمية الصوتي، ينقسم فيره الإقحام إلى ثلاثة:

a الإقحام البدئي، ويكون بإدخال صوت غير أصلي إلى المفردة في أولها، مثل لبز تحولت إلى بلبز، عفس تحولت إلى دعفس.

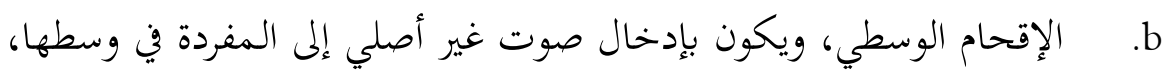
مثل لفظ درج تحول إلى دهرج، وبقر تحول إلى بيقر. c الإقحام الآخري، ويكون بإدخال صوت غير أصلي إلى المفردة في آخرها، مثل سمخ تحول إلى سمخر، وخلب إلى خلبس. 


\section{Uki Sukiman}

هو إحداث وحدات معجمية جديدة نتيجة ما يطرأ على الجذور. والقواعد الأساسية

$$
\text { المشهورة في التوليد الصرفي أربع، هي: }
$$

أ. الاشتقاق، هو صوغ وحدة معجمية ذات بنية صرفية بسيطة من أصل ما، وأهم ضروب

$$
\text { التولد الاشتقاقي التي تتيحها العربية ثلاثة عشر: }
$$

اشتقاق فعل من فعل، مثل عقلن على وزن فعلن، وتمسكن على وزن تمفعل.

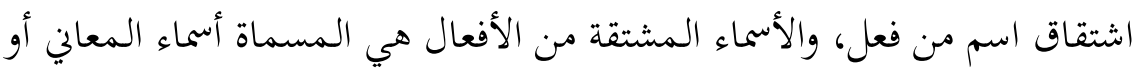

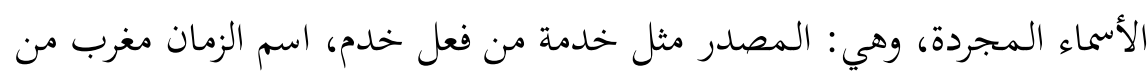
غرب، اسم المكان مسجد من سجد، اسم الآلة مضرب من ضرب، واسم المرة

$$
\text { ضربة من ضرب، واسم الهيئة جلسة من جلس. }
$$

اشتقاق صفة من فعل، ومن الصفات في العربية اسم الفاعل، اسم المفعول، والصفة

المشبهة، وأفعل التفضيل، وصيغ المبالغة والنسبة.

اشتقاق اسم من اسم، ويشتمل على :(1) المصادر الصناعية كإنسانية، وحيوانية،

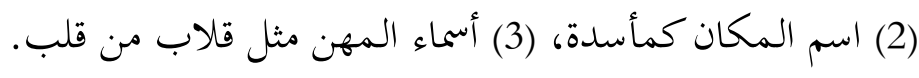

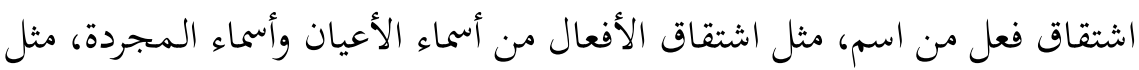

$$
\text { استنوق من اسم ناقة، وصعب ومن اسم صعب. }
$$

اشتقاق صفة من اسم: مثل اشتقاق اسم المفعول والنسبة من أسماء الأعيان وأسماء

$$
\text { المعاني، مثاله: مبرود من برد، حشراتي من حشر؟ }
$$

اشتقاق صفة من صفة: مثل اشتقاق النسبة من اسم الفاعل والصفة المشبهة،

وصيغة المبالغة، مثاله: مديري من مدير، وهاشمي من هاشم، وطليعي من طليعة. اشتقاق فعل من صفة، مثل تمسكن من مسكين، عقلاني من عقلن.

اشتقاق اسم من صفة، مثل اشتقاق المصدر الصناعي من اسم الفاعل واسم

$$
\text { المفعول، مثل عالمية من عالم، وقابلية من قابل. }
$$

اشتقاق صفة من ظرف: مثل اشتقاق النسبة من ظرف الزمان وظرف المكان،

$$
\text { مثاله: تحتاني من تحت وقبلي من قبل. }
$$




\section{Uki Sukiman}

$$
\text { اشتقاق فعل من أداة مثل سوف من سوف، وعنعن من عن. }
$$

اشتقاق اسم من أداة مثل اشتقاق المصدر الصناعي من الضمير واسم الموصول

$$
\begin{aligned}
& \text { وأداة الاستفهام، مثاله: كمية من كم، هوية من هو، ماهية من ما. } \\
& \text { (13 اشتقاق صفة من أداة مثل لامية من لام، إمع من مع. }
\end{aligned}
$$

ب. النحت، ويكون النحت بصوغ وحدة معجمية بسيطة من وحدتين معجميتين بسيطتين.

$$
\text { مثاله: عبدشمي من عبد + شمس، لاشى من لا+شيء. }
$$

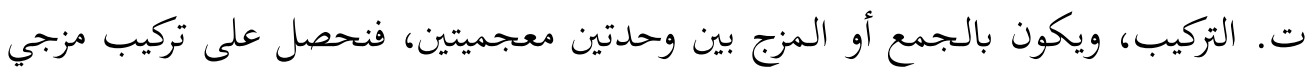

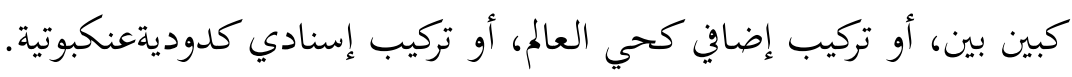

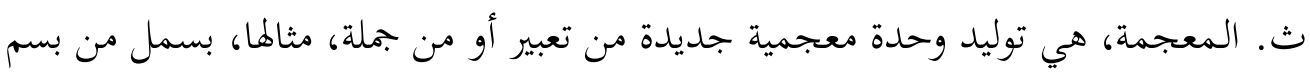
الله الرحمن الرحيم، وحوقل من لا حول ولا قوة إلا بالله.

(3) - (3) - (التوليد الدلالي

هو من أنواع قواعد التوليد في المعجم الذي يحصل عن التغيير الدلالي، وهذا النوع يشمل

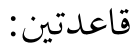

أ. التوليد بالمجاز أي أن ينتقل بوحدة معجمية ما من دلالتها الأصلية إلى دلالة جديدة، ويكون ذلك الانتقال إما توسيعا مثل "الكفر" وإما تضييقا مثل "السيارة." ب. الترجمة الحرفية هي ضرب من الاقتراض المعجمي، حيث ينتقل فيه المدلول دون الدال من لغة مصدر إلى لغة مورد، مثل التواصل الاجتماعي من أصل إنجليزي Social Network.

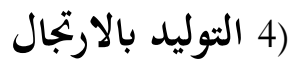
يكون باختلاق وحدة معجمية تتوفر فيها ثلاث خصائص تمييزية ضرورية، هي الانتماء المقولي، والتأليف الصوتي، والبنية الصرفية. ويمكن تصنيف هذا النوع من التوليد إلى نوعين: (1) الارتحال الحقيقي، هو باختلاق مفردة عامة أو مصطلح، مثل الشيفران، (2) الاربحال الاتباعي، وهو صوغ مفردة بالمحاكاة لمفردة أخرى أو باتباع لصيغة أخرى، مثل إني لآتية بالغدايا والعشايا. 


\section{Uki Sukiman}

هو توليد الوحدات المعجمية الجديدة اعتمادا مفردات من لغات أخرى. ينقسم هذا النوع من التوليد إلى قسمين: (1) الدخيل مثل ألمنيوم من aluminum، (2) المعرب مثل فلم منFilm . أما نصر الدين (Nasruddin 2017) في مقالته المنشورة في مجلة كلية الدراسات الإسلامية والعربية للبنات في دمنهور مصر فيقدم ست طرق التوليد، وهي:

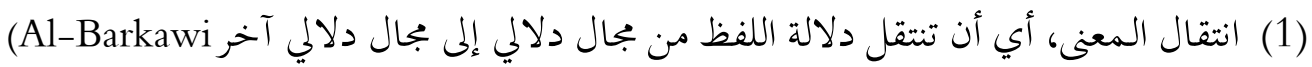
(2000. ويتم ذلك - كما أشار إليه خليل - عن طريق إعطاء لفظة عربية قديمة معنى مختلفا عما كان العرب يعرفونه بها (Khalil 1985a). وذلك مثل كلمة "الحكومة" التي كانت تعني في العصور القديمة الفصل بين المتخاصمين، فانتقل المعنى ليدل في الوقت الراهن على بجموعة الوزراء الذين يمثلون

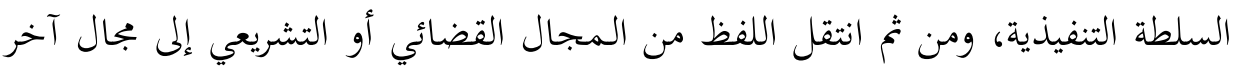
هو المجال التنفيذي. وكذلك كلمة بهلول التي كانت تعني السيد الجامع لكل خير والضاحك ثم أصبحت تدل على المهرج والمأفون. وكلمة الجدول كان معناها الأصل ولى النهر الصغير، ثم تدل على خطوط مستقيمة متقاطعة تحوي بعض البيانات. الاشتقاق، هو أن تشتق الكلمة من مادة عربية يعرفها العرب القدماء، ولكنهم لم يعرفوا الكلمة المشتقة ولا مدلولها، مثال ذلك الدبابة: من دب على الأرض، ومولده آلة من

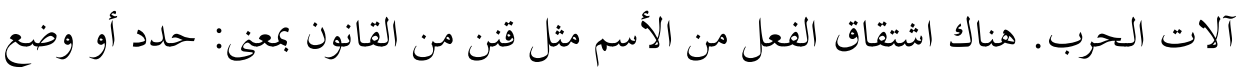
القانون، ومَوَّل من المال أي: قدم المال اللازم لمشروع مالـ

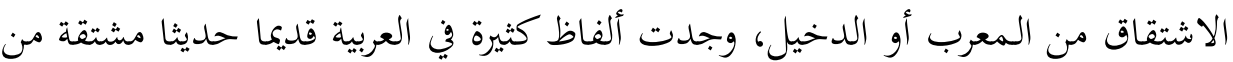

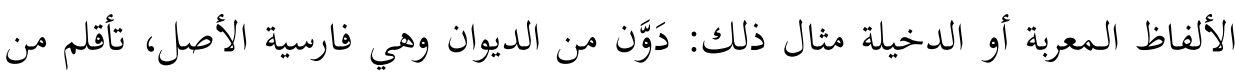
إقليم يونانية.

التوليد بالنحت والتركيب، ويتم ذلك عن طريق توليد كلمة منحوتة أو مركبة من حروف

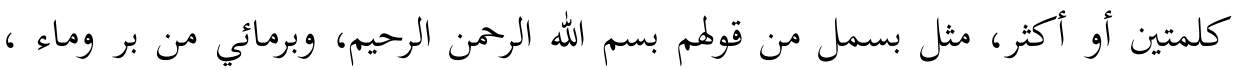
مولده من الحيوان يعيش في الماء والبر. وبجلس الشيوخ للدلالة على أحد بحالس البرمان 


\section{Uki Sukiman}

التوليد المجازي، يكون ذلك بتوليد اصطلاحات مركبة من الكلمات التي تدل مجازا على

بعض المعاني الخاصة مثل ضرب الرقم القياسي: ذهب بعيدا في النجاح أو الفشل. (6) التوسع في النسب مثل الطفيلي لغة محدثة. كان الرجل بالكوفة يقال له طفيل يأتي الولائم من غير أن يدعى إليها فنسب إليه (As-Suyuthi 1998) وما قدمه مراد من أنواع التوليد اللغوي وقواعد تكوينه يعد من أوسع البحوث عند رأي الكاتب، وخاصةعندما يتحدث عن طرق أوخطوات فيصيغ كلمات جديدة. ولكن وجد الكاتب الالتباس في تقسيمه التوليد الصرفي (Murad 1997). يشير مراد إلى أن المعجمة تختلف عن النحت، لأن المعجمة هي توليد وحدة معجمية جديدة من تعبير أو من جملة، بينما النحت هو توليد وحدة معجمية بسيطة من وحدتين معجميتين بسيطتين.

وماذكره مراد في كتابه يختلف عن بعض اللغويين العرب،مثل ما أشار إليه عبد القاهر بن مصطفى المغربي في كتابه الاشتقاق و التعريب (Al-Magribi 1947) حين يعرف النحت في الاصطلاح أن تعمد إلى كلمتين أو جملة فتنزع من مجموع حروف كلماقا كلمة فذة تدل على ما كانت تدل عليه الجملة نفسها. وهذا التعريف عند المغربي هو مما يعرفه أهل اللغة أنفسهم وجروا عليه في كلامهم، وفي المعاجم اللغوية شواهد كثيرة على ذلك.

يقول ابن فارس إن النحت هو أن تؤخذ كلمتان وتنحت منهما كلمة تكون آخذة منها جميعا، مثل حي على الرجل اذا قال حي على الرجل(Ibn Faris 1979). ويرى ابن فارس إلى أن النحت لا يتم فقط بوضع كلمة من كلمتين وقد يكون من ثلاث كلمات مثل النقرشة بمعنى الحس الخفي، وهي منحوتة من نقر وقرش ونقش. بناء على هذا، يرى الكاتب أن

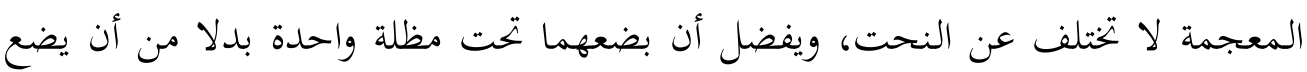
المعجمة في مكان و النحت في مكان آخر.

الحخلاصة

إن الدراسة في طرق التوليد اللغوي مهمة للغاية، لأها تعطينا صورة طويلة و واسعة عن أن اللغة العربية لديها قواعد وقوانين واضحة لاختلاف وحدة معجمية جديدة. وهذه القواعد 
Uki Sukiman

والقوانين بدورها تشير إلى قوة اللغة العربية وكفاءقا لمواجهة متطلبات الحياة. وترجع قوة اللغة العربية وكفاءتا إلى أن اللغة العربية تتمتع بمرونة عالية في توليد كلمات جديدة وتثبت أيضا أن اللغة العربية قادرة على التطور والبقاء بسبب مرونتها، وهي قادرة على مواجهة تحديات العصور المتنوعة. تستطيع اللغة العربية أن تتماشي مع الزمن بمستوياتا المختلفة، صوتية وصرفية ونوية ودلالية.

\section{المصادر والمراجع}

'Aid, M. 1980. Al-Madhahir At-Thariah 'ala Fusha-Lahn-Tashif-TaulidTa'rib-Mushtalah Ilmi. Kairo: Alim al-Kutub.

Al-Azhari, M. A. 2001. Tahdzib Al-Lugah. Edited by Muhammad Aud Mar'ab. 1st ed. Beirut: Dar Ihya Turats 'Arabi.

Al-Barkawi, A. F. 2000. Fi Dilalah Lughawiyah. Riyadh: Muassasah AlJaris Li Thaba'ah.

Al-Magribi, A. Q. .M. 1947. Al-Isytiqaq Wa At-Ta'rib. Kairo: Lajnah AtTa'lif wa At-Tarjamah wa An-Nasr.

Andriani, A. 2010. “Arabisasi Kosa-Kata Asing: Analisis Fonologi Dan Morfologi Pada Kosakata Serapan Dari Bahasa Inggris Dalam Kamus Al-Mawrid: Qamus 'Araby-Inkiliziy'." Yogyakarta: UIN Sunan Kalijaga.

Anis, I. 1958. Dalalah Alfadz. Kairo: Maktabah al-Anjalu al-Misriyah.

As-Suyuthi, J. 1998. Al-Mazhar Fi Ulum Al-Lugah Wa Anwa'uha. Beirut:

Dar Al-Kutub Al-Ilmiyah.

Chaer, A., and Leoni Agustina. 2010. Sosiolinguistik, Perkenalan Awal. Jakarta: PT Rineak Cipta.

Ibn Faris. 1979. Mu'jam Maqayis Al-Lugah. Beirut: Dar Fikr.

Khalil, H. 1985a. Al-Muwallad: Dirasah Fi Numuwwi Wa Tathawwur Al- 
Uki Sukiman

Lugah Al-Arabiyah Ba'da Al-Islam. Kairo: Al-Haiah al-Misriyah alAmmah lil Kitab.

- - . 1985b. Al-Muwallad: Dirasah Fi Numuwwi Wa Tathawwuri Lugah Arabiyah $B a^{\prime}$ da Islam. Kairo: Al-Haiah al-Misriyah al-Ammah lil Kitab.

Ibn Mandur. n.d. Lisan Al-Arab. Kairo: Dar Al-Ma'arif.

Mivtakh, B. A. 2019. “Arabic Language Neologism on the Field of Technology in Al-Akhbār Newspaper." Izdihār : Journal of Arabic Language Teaching, Linguistics, and Literature 2, no. 1: 17.

Murad, I. 1997. Muqaddimah Li Nadhariyah Al-Mu'jam. Tunisia: Dar AlGarib Al-Islami.

Nabila, A. 2017. “Penggunaan Kata Asing Dalam Iklan Elektronik Berbahasa Arab Di Website Exstra." UIN Sunan Kalijaga Yogyakarta.

Nasruddin, S.M. 2017. “Al-Muwallad Fi Al-Arabiyah Baina Rafd Wa Qabul." Majallah Kuliyah Ad-Dirasah Al-Islamiyyah Wa Al-Arabiyah Lil Banat Damanhur Misr, 2017.

Sukiman, U. 2020. “Neology of Arabic in Indonesia." Jurnal Al Bayan: Jurnal Jurusan Pendidikan Bahasa Arab 12, no. 2: 357. https:// doi.org/https:// doi.org/10.24042/albayan.v12i2.6071.

Tunji, M. 2005. Al-Mu'rab w Ad-Dakhil Fi Lugah Arabiyah. Beirut: Dar AlMa'rifah.

Wafi, A.A.W. 2014. Figh Lugah. Kairo: Dar Nahdhah Li Thab'i wa Nasr. 


\section{Uki Sukiman}

This Page Is Intentionally Left Blank تركت هذه الصفحة فارغة عمدا

'Halaman Ini Sengaja Dikosongkan' 